\title{
PID CONTROLLER TUNING SCHEME FOR TWIN ROTOR MULTI-INPUT MULTI-OUTPUT SYSTEM BASED PARTICLE SWARM OPTIMIZATION APPROACH
}

\author{
El-Sayed M. Ahmed, and M. Abd-Elhady Mohamed \\ Computers and Systems engineering Department Minia University
}

(Received May 15, 2009 Accepted May28, 2009).

\begin{abstract}
This paper presents a new intelligent control scheme which utilizes particle swarm optimization (PSO) for off-line tuning of proportionalintegral-derivative (PID) controller for the twin rotor multi-input multioutput system (TRMS). The control objective is to make the beam of the TRMS move quickly and accurately to the desired attitudes. The TRMS exhibits MIMO characteristics, high order non-linearity, significant cross coupling and inaccessibility of some of its states and outputs for measurements. PSO Algorithm is successfully implemented to this problem. Experimental and simulated results of the developed PID controller for a twin rotor system are given to demonstrate its effectiveness. Satisfactory results are anticipated in the experimental as well as in the simulation results. In an attempt to evaluate the performance of the developed controller an experimental and simulated comparative assessments with the conventionally PID tuned method (Ziegler-Nichols method) has been conducted. The results of the PID controller based PSO reveals better performances indices than the other conventional controller.
\end{abstract}

KYWORDS -Evolutionary Computing; Particle swarm algorithm; PID controller; TRMS

\section{INTRODUCTION}

In recent years, optimization algorithms have received increasing attention by the research community as well as the industry to solve various complex control problems as an alternative or complement to the conventional methods [1]. Optimization techniques using analogy of swarming principle have been adopted to solve a variety of engineering problems in the past decade. Swarm Intelligence (SI) is an innovative distributed intelligent paradigm for solving optimization problems that originally took its inspiration from the biological examples by swarming, flocking and herding phenomena in vertebrates. A population of particles exists in the n-dimensional search space in which the optimization problem lives in. each particle has a certain amount of knowledge, and will move about the search space based on this knowledge. The particle has some inertia attributed to it and so it will continue to have a component of motion in the direction it is moving [2].

Parsopoulos and Vrahatis attempted to improve the search efficiency in PSO by performing two stage transformation of the objective function which eliminates and elevates the neighborhood of the local minima [3]. Alternative runs and tumbles in 
Ecoli bacteria found in the human intestine constitute chemo taxis and this foraging mechanism was imitated by Kevin Passino for solving optimization problem in control system [4]. In the earlier PSO algorithms, each particle of the swarm is accelerated by its best previous position and towards the best particle in the entire swarm. Here, the underlying assumption is that each particle in the swarm remembers the best position already visited and also it is informed about the best particle position. After letting the particles to search adequate number of times in the solution space independently for the best possible positions, they are attracted to the basin containing the best particle by establishing proper communication among them about the search environment [5].

Genetic Algorithm also considered the famous evolutionary tuning method which has been implemented in twin rotor modeling and controller parameter tuning through recent literature. Although GA can provide good solutions in tuning controllers that has a complex model, it is requires huge memory and faster processing units with large word lengths to execute huge number of repeated computations. Moreover, for highly multi-modal problems, the solutions may lose diversity and get trapped in local minima at some points unless special method is adopted to avoid premature convergence to suboptimal region of the search space

The purpose of this research is to investigate, implement and evaluate an optimal PID controller design using, Particle swarm optimization techniques for TRMS process. In an attempt to evaluate the performance of the developed controller adopting (PSO) algorithm, a comparison study of the Experimental results of using other two methods for the tuning of PID controllers for TRMS has been conducted. One method is using particle swarm optimization for the tuning of PID-controller while the other is using Ziegler-Nichols technique for the same purpose. This paper is organized as follows. Section II gives a description of The Lab-scale TRMS, while Section III gives a PID controller overview. The PSO Algorithm is presented in section IV. Simulation results and experimental verifications of PID controller adopting PSO is provided in section V. A comparative assessment of the proposed PID-controller and ZieglerNichols technique for the same purpose is concluded in section VI. Finally conclusion of this work is presented in section VII

\section{TWIN ROTOR MIMO MATHEMATICAL MODEL}

\section{A. System Description}

A scaled and simplified version of a practical helicopter, namely the twin rotor system (TRMS) is often used as a laboratory platform for control experiments. This system resembles a helicopter in many aspects. Since the TRMS permits both 1 and 2 degrees of freedom (DOF) motions, it can be considered as a static test rig for an air vehicle [1]. The schematic of the Lab-scale, twin- rotor, multiple input-multiple output (MIMO) system is shown in Fig. 1. 


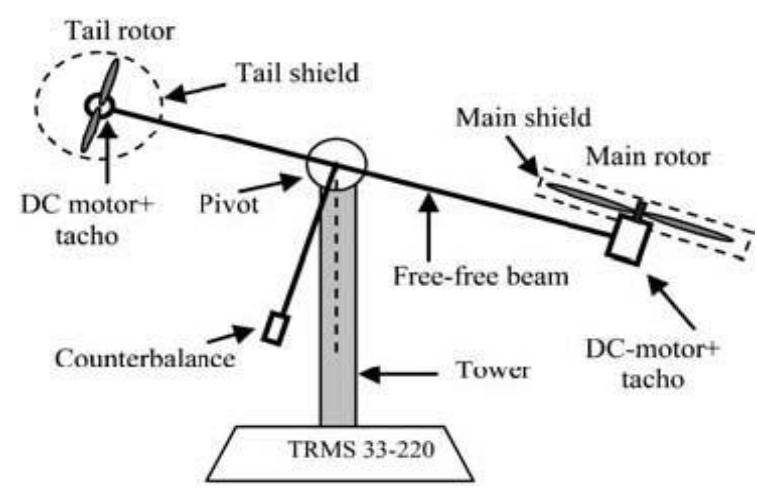

Fig. 1 The prototype of Lab-scale helicopter system

The Lab-scale Helicopter consist of a beam pivoted on its base in such a way that it can rotate freely in both its horizontal and vertical planes. There are two rotors (the main and tail rotor), driven by DC motors, at each end of the beam. Either or both axes of rotation can be locked by means of two locking screws provided for physically restricting the horizontal or vertical plane of the beam. The joined beam can be moved by changing the input voltage to control the rotational speed of these two propellers. There is a pendulum counter-weight hanging on the joined beam which used for balancing the angular momentum in steady state or the load. In cretin aspects its behaviour resembles that of a helicopter. it is difficult to design a suitable controller because of influence between two axes and nonlinear movement. From the control point of view, it exemplifies a high order nonlinear system with significant cross coupling. The mathematical model of Lab-Scale Helicopter we employ here is discussed in [6] and [8]. Fig.2 is shown the system acting effective forces in the vertical plane. The physical variables as well as the symbol definitions along with an overview of the mathematical model derivation and its dynamic equations are presented below.

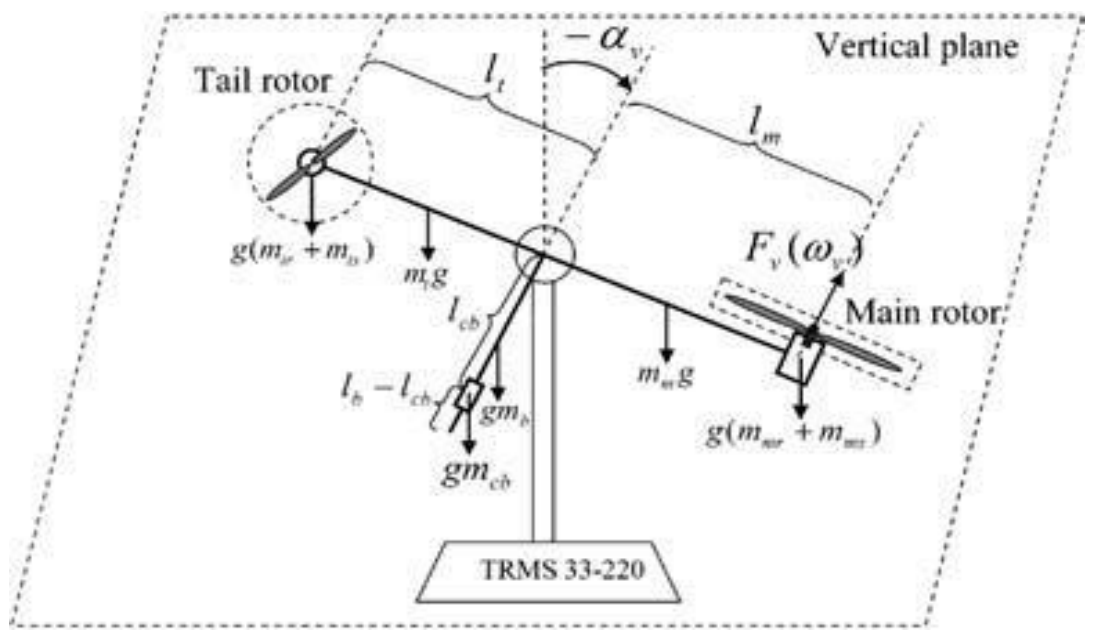

Fig. 2 Effective forces in vertical plane 


\section{B. Notation \\ C. Notation}

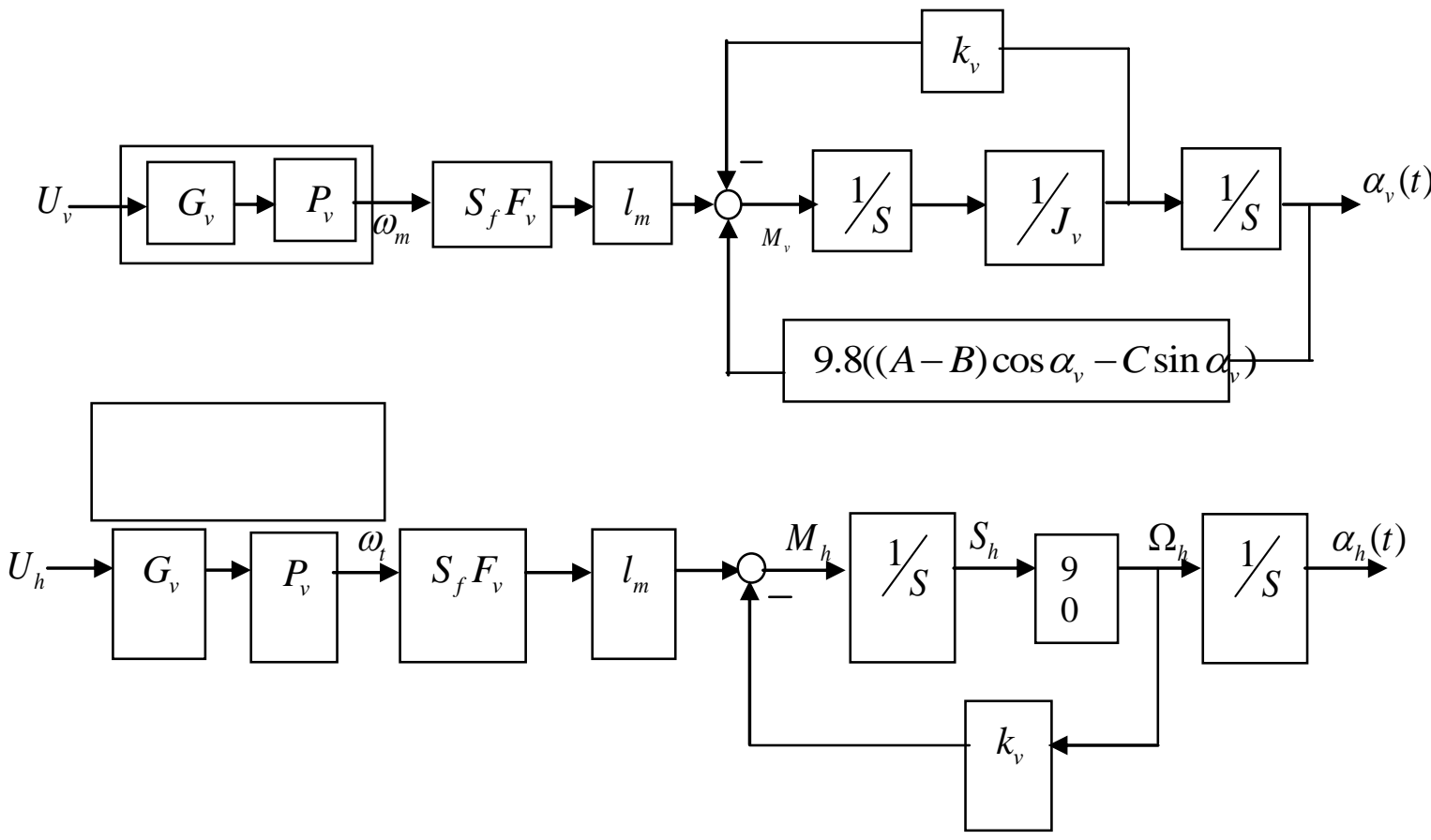

Fig. 3 Block diagram of decoupled twin rotor system

A block diagram of TRMS model is shown in Figure 3. The equation of each mathematical block diagram will clarify as following:

\section{Main Rotor Model}

$$
\begin{aligned}
& \frac{d S_{v}}{d t}=l_{m} S_{f} F_{v}\left(w_{m}\right)-\mathrm{W}_{v} k_{v}+g\left((A-B) \operatorname{cod} a_{v}-C \sin a_{v}\right)-0.5 \mathrm{~W}_{h}^{2}(A+B+C) \sin 2 a_{v} \\
& \frac{d a_{v}}{d t}=\mathrm{W}_{v} \\
& \mathrm{~W}_{v}=\frac{S_{v}+J_{t r} w_{t}}{J_{v}} \\
& \frac{d u_{v v}}{d t}=\frac{1}{T_{m}}\left(-U_{v v}+U_{v}\right) \\
& w_{m}=P_{v}\left(U_{v v}\right)
\end{aligned}
$$

where

$$
A=\left(\frac{m_{t}}{2}+m_{t r}+m_{t s}\right) l_{t} \quad B=\left(\frac{m_{m}}{2}+m_{m r}+m_{m s}\right) l_{m} \quad C=\left(\frac{m_{b}}{2} l_{b}+m_{c b} l_{c b}\right)
$$




\section{Tail Rotor Model}

$$
\begin{aligned}
& \frac{d S_{h}}{d t}=l_{t} S_{f} F_{h}\left(w_{t}\right) \cos a_{v}-\mathrm{W}_{h} k_{h} \\
& \frac{d a_{h}}{d t}=\mathrm{W}_{h} \\
& \mathrm{~W}_{h}=\frac{S_{h}+J_{m r} w_{m} \cos a_{v}}{J_{h}}=\frac{S_{h}+J_{m r} w_{m} \cos a_{v}}{D \sin ^{2} a_{v}+E \cos ^{2} a_{v}+F}
\end{aligned}
$$

Assume the tail rotor is an independent system then (6) to (8) can be written as:

$$
\begin{aligned}
& \frac{d S_{h}}{d t}=l_{t} S_{f} F_{h}\left(w_{t}\right)-\mathrm{W}_{h} k_{h} \\
& \frac{d a_{h}}{d t}=\mathrm{W}_{h} \\
& \mathrm{~W}_{h}=90 S_{h}
\end{aligned}
$$

The horizontal motion of the beam (around the vertical axis) can be described as a rotational motion of a solid mass it should. Be noted that angular velocities are non-linear functions of the input voltage of the DC-motor. Thus, we have two additional set of equations.

\section{E. The Nonlinear Equation of Main Motor}

$$
\begin{aligned}
& \frac{d u_{v v}}{d t}=\frac{1}{T_{m r}}\left(-U_{v v}+U_{v}\right) \\
& \omega_{m}=P_{v}\left(U_{v v}\right) \\
& \frac{d u_{h h}}{d t}=\frac{1}{T_{t r}}\left(-U_{h h}+U_{h}\right) \\
& \omega_{t}=P_{h}\left(U_{h h}\right)
\end{aligned}
$$

The above model of the motor-propeller dynamics is obtained by substituting the nonlinear system by a serial connection of a linear dynamic system and static nonlinearity [6].

\section{PID CONTROLLER}

Despite the many sophisticated control theories and techniques that have been devised in the last few decades, PID controllers continue to be the most commonly used in the industrial processes. These controllers have a simple structure and are easy to be implemented by the great majority of industrial practitioners and automatic control designers. They are used in processes whose dynamics models can be described as first or second-order systems. In practice, most physical systems have inherently intractable characteristics such as high order and non-linearties [7]. 
In general, a transfer function $\mathrm{G}_{\mathrm{c}}(\mathrm{s})$ of single loop PID controllers has the following form:

$G_{c}(s)=\frac{u(s)}{e(s)}=K_{p}+\frac{K_{I}}{s}+K_{D} s$

Where e(s) is the error signal, which is the difference between the system input $\mathrm{u}(\mathrm{s})$ and output, $\mathrm{K}_{\mathrm{p}}, \mathrm{K}_{\mathrm{i}}$ and $\mathrm{K}_{\mathrm{d}}$ are proportional, integral and derivative gains, respectively. The problem is to determine values of gins so that performance requirements are satisfied. However, for an MIMO system with complicated dynamics, it is difficult to design an appropriate PID controller by using the conventional control theory. The manner of obtaining the parameters of PID controllers for MIMO systems that satisfy a certain system performance requirement has been addressed in many studies [8-9].

\section{A. The Optimization Problem}

The MIMO PID controller design problem can be formulating as an optimization and search problem as follows [11]:

$f: \mathrm{S} \rightarrow \mathfrak{R}$

Where $\mathrm{S}$ is the set of solutions and best choices are that for the function $\mathrm{f}$ is optimal. In the context of controller, a candidate system can be represented by a uniform parametric vector given by

$$
s_{i}=\left\{g_{1}, g_{2}, \ldots . ., g_{n}\right\} \in \mathfrak{R}
$$

Where $\mathrm{i}$ stand for the $i^{\text {th }}$ possible candidate solution, $\mathrm{n}$ the number of parameters required by the solution, $g_{j} \in \mathfrak{R}$ the $j^{\text {th }}$ parameter of the $i^{\text {th }}$ candidate solution with $j \in\{1, \ldots, n\}$, and $\mathfrak{R}^{n}$ the n-dimensional real Euclidean space. We have an objective function $\mathrm{Q}(\mathrm{S})$ needs to be optimized. Where $\mathrm{Q}(\mathrm{S})$ represents the quality measurement for a solution $\mathrm{S}_{\mathrm{i}}$ given $\forall Q\left(S_{i}\right)>0$. The problem is to find the best solution (i.e., controller) such that:

$Q(\tilde{s})=\operatorname{Min}_{S} Q(s)$

Two error signals are formed for each channel taking the difference between the desired and actual Output

$e_{v}(t)=\alpha_{v r}(t)-\alpha_{v}(t)$

$\left.e_{h}(t)=\alpha_{h r}(t)-\alpha_{h}(t)\right\}$

where $\alpha_{\mathrm{v}}(\mathrm{t})$ and $\alpha_{\mathrm{h}}(\mathrm{t})$ are the measured output for vertical and horizontal positions while, $\alpha_{\mathrm{vr}}(\mathrm{t})$ and $\alpha_{\mathrm{hr}}(\mathrm{t})$, are the required output for vertical and horizontal positions respectively. Both of these error signals $e_{\mathrm{v}}(\mathrm{t})$ and $e_{\mathrm{h}}(\mathrm{t})$ are used to formulate the objective function, $f(t)$, of the optimization process. In this work, sum of mean absolute error is chosen as the objective

$f(t)=\sum_{t=1}^{N}\left[e_{v}(t)^{2}+e_{h}(t)^{2}\right]$ 


\section{B. MIMO PID Controller Configuration}

The basic configuration of PID controllers of the MIMO system is shown in Figure 3. Where $r(t)$ is the controller set point vector, e $(t)$ is the error signal vector, $u(t)$ is the controller output vector, and $\mathrm{y}(\mathrm{t})$ is the process output vector respectively.

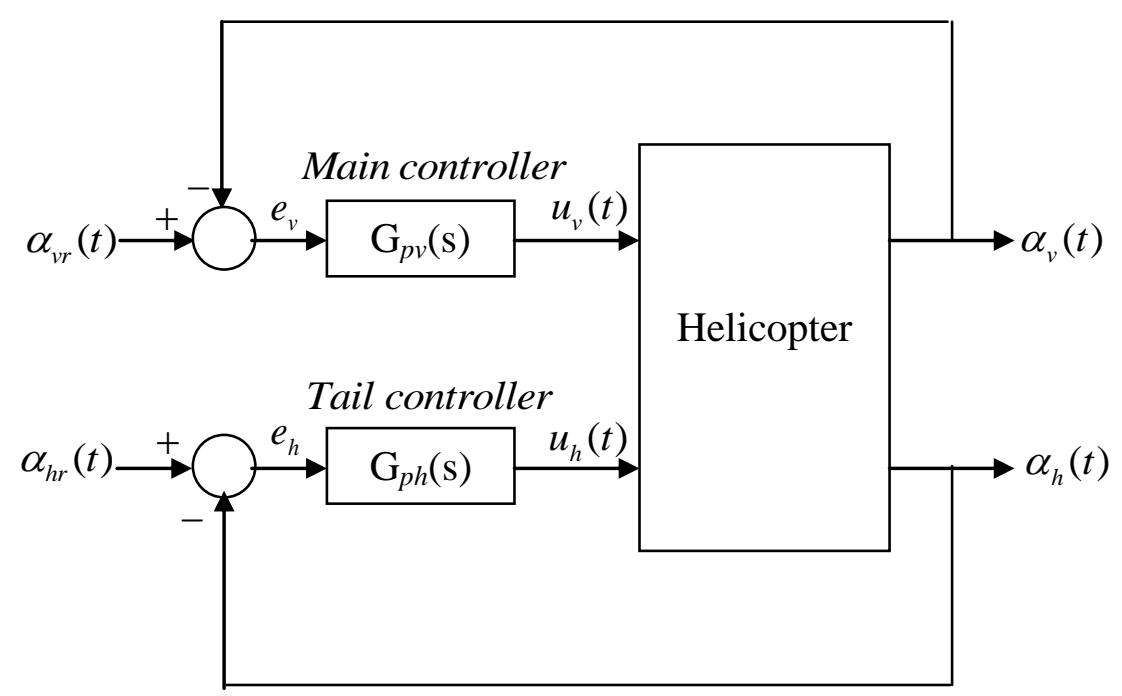

Fig. 4 The basic configuration of MIMO PID Controller

\section{Ziegler-Nichols approach}

The well-known method, Ziegler-Nichols method, provides a systematic PID tuning approach. This method has experienced good load disturbance attenuation in many cases. however, it have severe drawbacks, they use insufficient process information and the design criterion gives closed loop systems with poor robustness [11] Ziegler and Nichols developed their tuning rules by simulating a large number of different processes, and correlating the controller parameters with features of the step response. The key design criterion was quarter amplitude damping. Process dynamics was characterized by two parameters obtained from the step response. For improving a certain system performance, e.g., rise time, overshoot, and integral of the absolute error, many studies are attempting to incorporate features on the basis of the experiences of experts with regards to PID parameters selection [12].

\section{PARTICLE SWARM ALGORITHM}

The particle swarm optimization PSO algorithm we employ here is discussed in [10]. PSO are based on two socio-metric principles. Particles fly through the solution space and are influenced by both the best particle in the particle population and the best solution that a current particle has discovered so far. The best particle in the population is typically denoted by (global best), while the best position that has been visited by the current particle is donated by (local best). The (global best) individual conceptually connects all members of the population to one another. That is, each particle is influenced by the very best performance of any member in the entire population. 
The (local best) individual is conceptually seen as the ability for particles to remember past personal success. The particle swarm optimization makes use of a velocity vector to update the current position of each particle in the swarm. The position of each particle is updated based on the social behaviour that a population of individuals adapts to its environment by returning to promising regions that were previously discovered [2].

Let the $i^{\text {th }}$ particle of the swarm is represented by the D-dimensional vector $x_{i}=\left(x_{i 1}, x_{i 2}, \ldots, x_{i D}\right)$ and the best particle in the swarm, i.e. the particle with the smallest function value, is denoted by the index g. The best previous position (the position giving the best function value) of the $i^{\text {th }}$ particle is recorded and represented as $p_{i}=\left(p_{i 1}, p_{i 2}, \ldots, p_{i D}\right)$, and the position change (velocity) of the $i^{t h}$ particle is $v_{i}=\left(v_{i 1}, v_{i 2}, \ldots, v_{i D}\right)$. The particles are manipulated according to the equations

$$
\begin{aligned}
& v_{i d}=w \cdot v_{i d}+c_{1} \cdot r_{1} \cdot\left(p_{i d}-x_{i d}\right)+c_{2} \cdot r_{2} \cdot\left(p_{g d}-x_{i d}\right) \\
& x_{i d}=x_{i d}+v_{i d}
\end{aligned}
$$

where $\mathrm{d}=1,2, \ldots, \mathrm{D} ; \mathrm{i}=1,2, \ldots, \mathrm{N}$ and $\mathrm{N}$ is the size of population; $\mathrm{w}$ is the inertia weight; $c_{1}$ and $c_{2}$ are two positive constants; $r_{1}$ and $r_{2}$ are two random values in the range $\{0,1\}$. The first equation is used to calculate $i^{\text {th }}$ particle's new velocity by taking into consideration three terms: the particle's previous velocity, the distance between the particle's best previous and current position, and, finally, the distance between swarm's best experience (the position of the best particle in the swarm) and $i^{\text {th }}$ particle's current position. Then, following the second equation, the $i^{\text {th }}$ particle flies toward a new position. In general, the performance of each particle is measured according to a predefined fitness function, which is problem dependent. The role of the inertia weight $\mathrm{w}$ is considered very important in PSO convergence behaviour. The inertia weight is employed to control the impact of the previous history of velocities on the current velocity. In this way, the parameter w regulates the trade-off between the global (wide-ranging) and local (nearby) exploration abilities of the swarm. A large inertia weight facilitates global exploration (searching new areas); while a small one tends to facilitate local exploration, i.e. fine-tuning the current search area. A suitable value for the inertia weight $\mathrm{w}$ usually provides balance between global and local exploration abilities and consequently a reduction on the number of iterations required to locate the optimum solution. A general rule of thumb suggests that it is better to initially set the inertia to a large value, in order to make better global exploration of the search space, and gradually decrease it to get more refined solutions, thus a time decreasing inertia weight value is used. The main steps of the PSO algorithm are shown in Figure 5. 


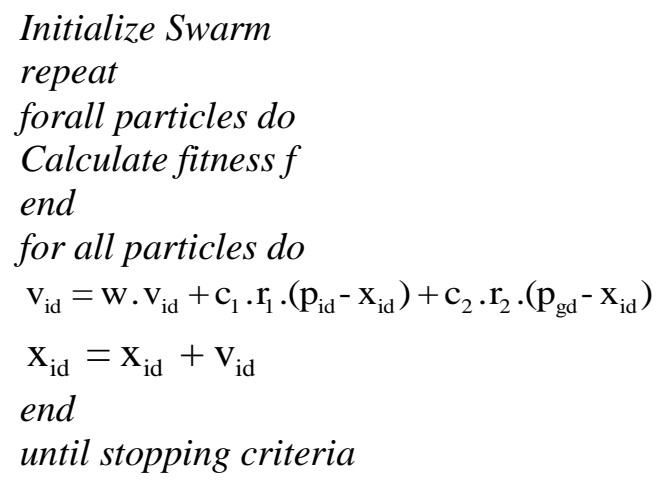

Fig. 5 Particle Swarm Optimization Pseudo Code

\section{SIMULATION RESULTS AND EXPERIMENTAL VERIFICATIONS}

\section{A. Simulation Results of PSO controller}

The PSO are applied to search the optimal parameters of PID controllers for the main rotor and the tail rotor respectively, in order to obtain the minimum error when the helicopter moves between two workspace points. To validate the design approach a completer Simulink model has been developed for this purpose. The PSO algorithm begins with a population of real numbers, called swarm. Each row represents a solution set, called particle. A swarm of hundred particles each particle consists of six elements (gains) each, i.e., $100 \times 6$ is created randomly within the range of $[0,20]$. The first three elements of each individual are normalized and assigned to the main rotor PID gains while the remaining three elements are normalized and assigned to the tail rotor PID gains respectively. The acceleration coefficients $c_{1}$ and $c_{2}$ were initially set to 0.5 whereas the inertia coefficient, $\omega$, was gradually decreased from 1.0 to 0.1 with generation. At the end of optimization process, six parameters representing the controller gain that satisfied the constraint in terms of stability and yielded minimum value in the objective function.

The obtained gains by PSO and Z-N methods have been verified by simulation as shown in Figures 6, 7, 8, and 9. Figure 6 and 7 are showing the tracking output of main rotor and tail rotor due to step input with final value 1.0 respectively. While, Figure 8 and 9 are showing the tracking output of main rotor and tail rotor due to sinusoidal input with amplitude 1.0 and frequency of $0.1 \mathrm{~Hz}$ respectively. It is observed from the system's response that the output trajectories of the main rotor and the tail rotor can perfectly follow the reference signals.

\section{B. Experimental Results and comparative assessments}

To validate the proposed design approach of the PID Controller tuning Scheme using PSO, the optimal values obtained from simulations has been applied in the on-line experiments of the prototype twin- rotor MIMO system. Moreover, the on-line experiments results has been compared to those obtained from the Ziegler-Nichols traditional technique. Figure 10 compares the outputs of main rotor of the Ziegler- 
Nichols tuned designed controller with the PID tuned controller by the PSO due to pulse input with final value 1.0 and frequency $0.025 \mathrm{~Hz}$. The associated cross coupling effect on tail rotor are shown in Figure 12. With the same respect, Figure 11 compares the outputs of tail rotor of the Ziegler- Nichols tuned designed controller with the PID tuned controller by the PSO due to pulse input with final value 1.0 and frequency 0.025 Hz. The associated cross coupling effect on the main rotor are shown in Figure 13. Table 2 and 3 are describeing the performance indices of system response adopting each of the controllers in terms of percentage overshoot, settling time, rise time and steady state errors along with each controller gain values.

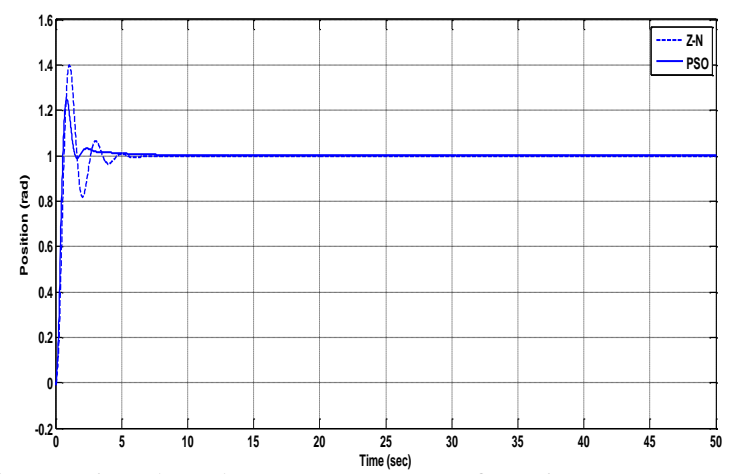

Fig. 6 Simulated step response of Main rotor Using PID tuned by PSO and Z-N

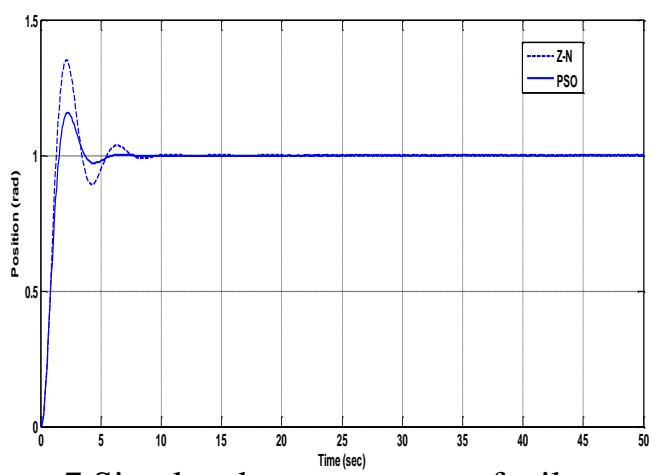

7 Simulated step response of tail rotor Using PID tuned by PSO and Z-N

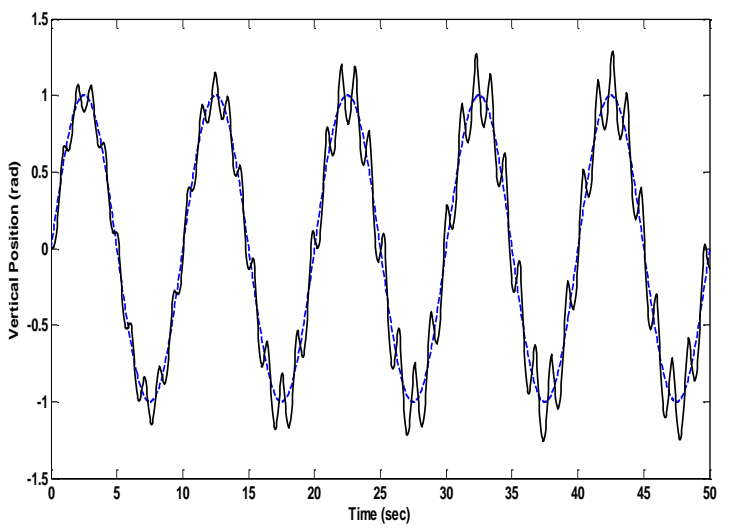

Fig. 8 Simulated tracking output of tail rotor of Main rotor using PID tuned controller by the PSO

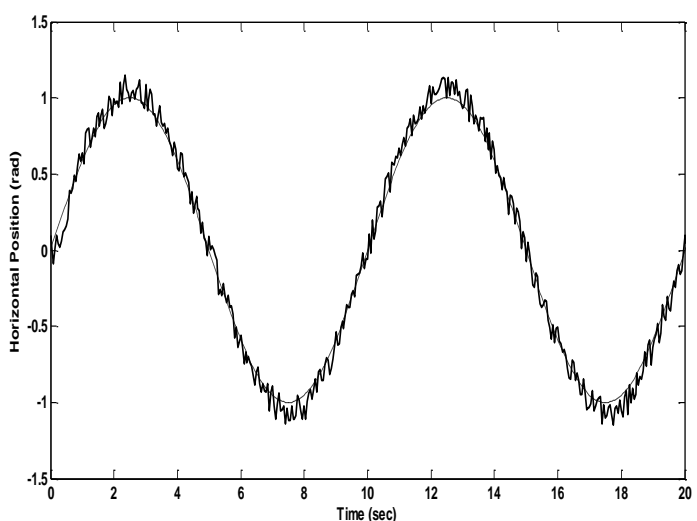

Fig. 9 Simulated tracking output of tail rotor using PID tuned controller by PSO 


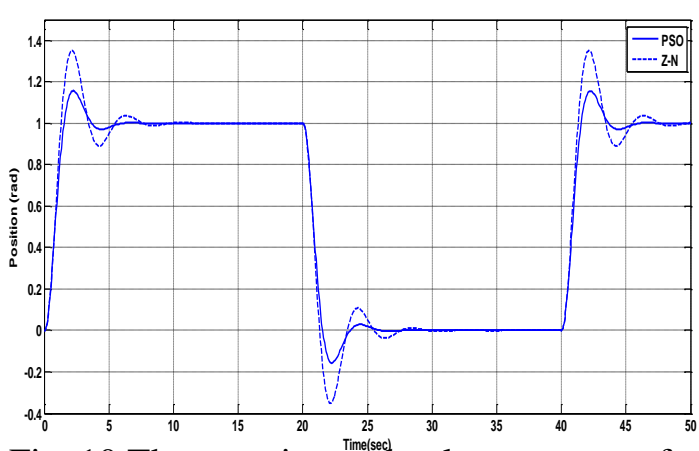

Fig. 10 The experimental pulse response of main rotor using PID tuned by PSO compared with Z-N controlleer

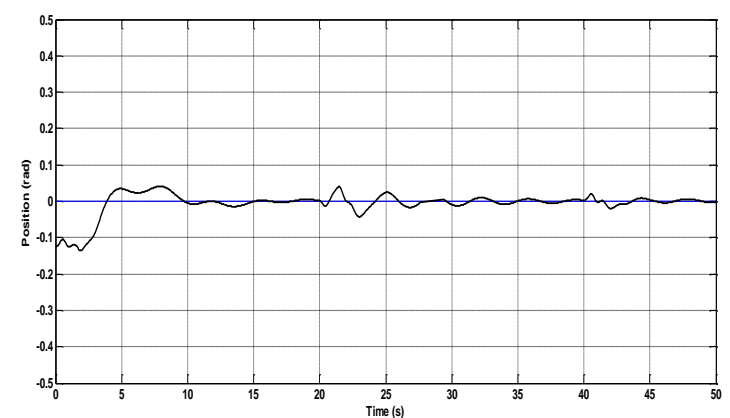

Fig. 12 The cross-coupling effect affected on the tail rotor due to main rotor controller action

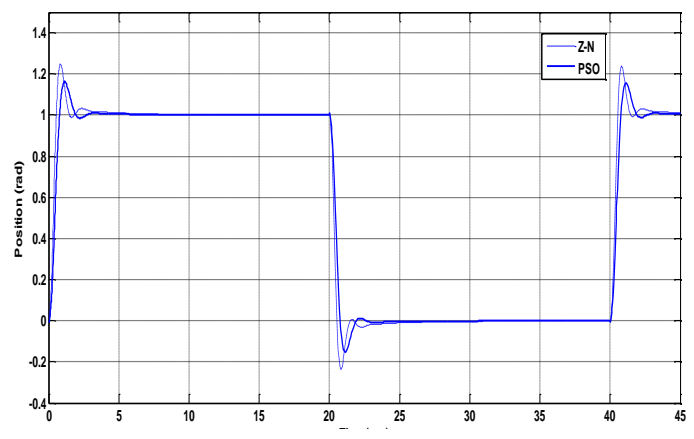

Fig. 11 The experimental pulse response of tail rotor using PID tuned by PSO comparedwith Z-N controller

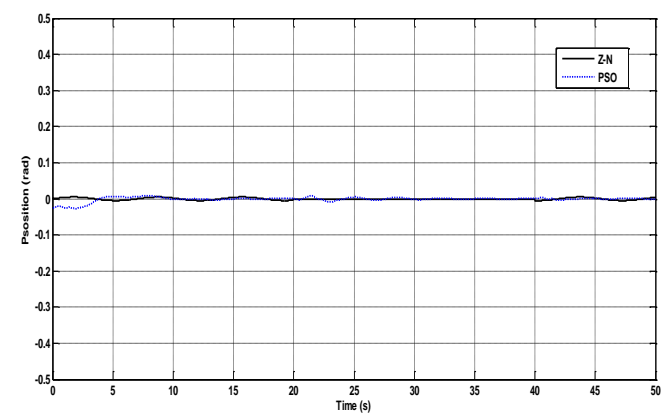

Fig. 13 The cross-coupling effect affected on the main rotor due to tail rotor controller action

TABLE II

Terms System Performance Indices Using PID with Particle swarm Optimization

\begin{tabular}{|c|c|c|c|c|c|c|c|}
\hline \multirow{2}{*}{ Symbol } & \multicolumn{7}{|c|}{ Particle swarm optimization } \\
\hline & $\mathrm{K}_{\mathrm{p}}$ & $\mathrm{K}_{\mathrm{i}}$ & $\mathrm{K}_{\mathrm{d}}$ & $\mathrm{T}_{\mathrm{s}}$ & $\mathrm{T}_{\mathrm{r}}$ & P.O \% & $\mathrm{e}_{\mathrm{ss}}$ \\
\hline Main Rotor & 11.78 & 11.7 & 5.26 & 3.65 & 1.54 & 21.47 & 0 \\
\hline Tail Rotor & 12.58 & 3.64 & 4.79 & 5.1 & 1.01 & 16.38 & 0 \\
\hline
\end{tabular}

TABLE III

TERMS System Performance Indices Using PID with Z-N

\begin{tabular}{|c|c|c|c|c|c|c|c|}
\hline Symbol & \multicolumn{7}{|c|}{ Zigler-Nicholas } \\
\hline & $\mathrm{K}_{\mathrm{p}}$ & $\mathrm{K}_{\mathrm{i}}$ & $\mathrm{K}_{\mathrm{d}}$ & $\mathrm{T}_{\mathrm{s}}$ & $\mathrm{T}_{\mathrm{r}}$ & P.O \% & $\mathrm{e}_{\mathrm{ss}}$ \\
\hline Main Rotor & 9.7 & 1.21 & 6.76 & 4.9 & 1.54 & 40.01 & 0 \\
\hline Tail Rotor & 8.2 & 1.01 & 7.23 & 7.3 & 1.15 & 36.73 & 0 \\
\hline
\end{tabular}




\section{CONCLUSION AND REMARKS}

In this paper an off-line PID controller parameters are optimally designed by the PSO algorithm then it has been verified through Simulink simulation as well as experimental work for Lab-scale twin rotor MIMO system.. The process under study perceived as a challenging control engineering problem owing to its MIMO characteristics, high order non-linearity, significant cross coupling and inaccessibility of some of its states and outputs for measurements. PSO based tuning methods have proved their excellence in giving better results by improving the steady state characteristics and performance indices. The proposed PSO tuned controller shows better performance criteria comparable with Ziegler-Nichols tuned controller.

\section{REFERENCES}

[1] M.O. Tokhi and M.S. Alam " Particle Swarm Optimization Algorithms and Their Application to Controller Design for Flexible Structure system".

[2] Nadia Nedjah," Swarm Intelligent Systems", Studies in Computational Intelligence, Springer, vol 26

[3] K.E. Parsopoulos and M.N. Vrahatis. Recent approaches to global optimization problems through particle swarm optimization. Natural Computing 1: $235-306$, 2002.

[4] Passino K.M. "Biomimicry of Bacterial Foraging for Distributed Optimization and Control", IEEE Control Systems Magazine, Vol. 22, No. 3, pp. 52 - 67, June 2002.

[5] S. Easter Selvan, Sethu Subramanian and S. Theban Solomon " Novel Technique for PID Tuning by Particle Swarm Optimization"

[6] Feedback Co., Twin rotor MIMO system user manual,1998.

[7] Wei-Der Chang, "A multi-crossover genetic approach to multivariable PID controllers tuning", Expert Systems with Applications 33 (2007) 620-626

[8] Wei-Yen Wang, Tsu-Tian Lee "Evolutionary Design of PID Controller for Twin Rotor Multi-Input Multi-Output System", Proceedings of the 4th World Congress on Intelligent Control and Automation, 2002, Shanghai, P.R.China

[9] Mohammed El-Said El-Telbany "Employing Particle Swarm Optimizer and Genetic Algorithms for Optimal Tuning of PID Controllers: A Comparative Study" ICGST-ACSE Journal, Volume 7, Issue 2, November 2007

[10] Kennedy J., and Spears M., "Matching Algorithms to Problems: An Experimental Test of the Particle Swarm and Some Genetic Algorithms on the Multimodal Problem generator", Proceedings IEEE International Conference of Evolutionary Computation, 1998.

[11] Yang Quan Chen, Moore, K.L. "Relay feedback tuning of robust PID controllers with iso-damping property" IEEE Trans Syst Man Cybern B Cybern. 2005 Feb; 35(1):23-31.

[12] Tore Hägglund, Karl Johan Åström "Revisiting the Ziegler-Nichols step response method for PID control" Journal of Process Control,14:6, pp. 635--650, 2004 


\section{"تنغيم متحكم تفاضلى تكاملى تتاسبى باستخدام اسلوب أسراب الجسيمات المثلى لطائرة}

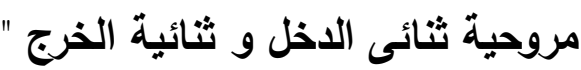

مدرس بجامعه المنيا قسم هندسة الحاسبات والنظم

معبد بجامعه الدنيا قسم هندسة الحاسبات والنظم

$$
\text { م. محمد عبدالهادى /حد محد }
$$

يتتاول هذا البحث استخادم احد طرق الذكاء الاصطناعى لتنغيم متحكم تتاسبى تفاضلى تكاملى

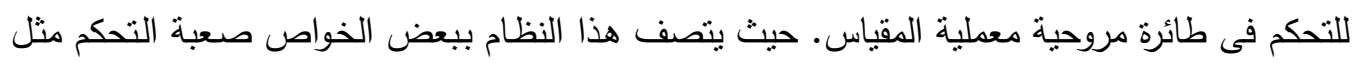

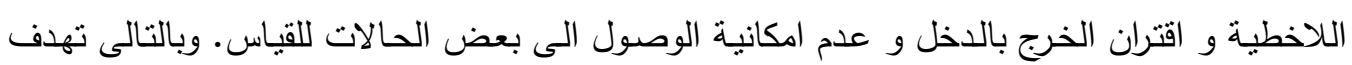

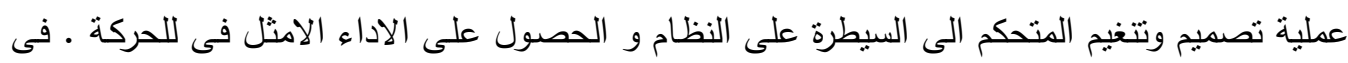

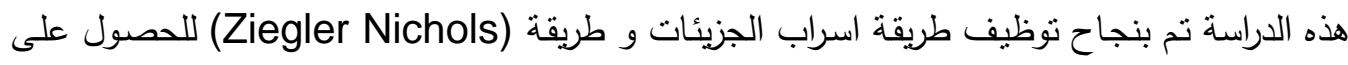

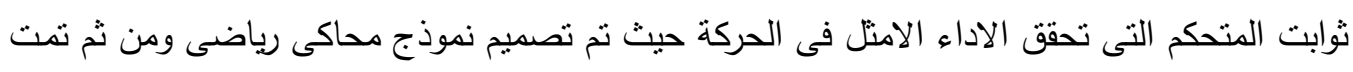

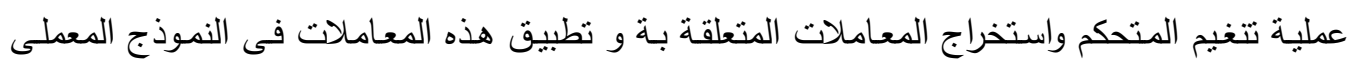

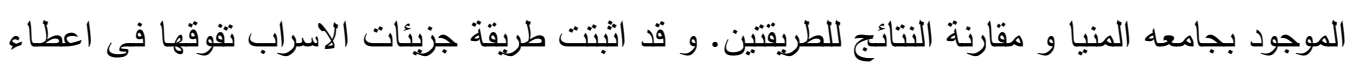
نتائج مرضية لتجارب الاداء 